Diabetologe 2009 · 5:336-337

DOI 10.1007/s11428-009-0463-4

Online publiziert: 1. Juli 2009

(c) Springer Medizin Verlag 2009

\author{
M. Haslbeck ${ }^{1,2}$ \\ ${ }^{1}$ Forschergruppe Diabetes e. V. am Helmholtz-Zentrum \\ München-Neuherberg, Neuherberg \\ ${ }^{2}$ Internistische Praxisgemeinschaft, München
}

\title{
Diabetische Neuropathie - eine Herausforderung für die Basiswissenschaften und die Suche nach Komorbiditäten
}

Im ersten, im Dezember 2008 erschienenen Themenheft „Diabetische Neuropathie I“ wurde in fünf Beiträgen über aktuelle, ausgewählte klinische Themen des großen Spektrums neuropathischer Krankheitskomplikationen bei Diabetes mellitus berichtet. Hierbei wurden insbesondere klinische Erscheinungsbilder sowie praktisch-diagnostische und therapeutische Möglichkeiten der sensomotorischen Neuropathie - dem häufigsten Manifestationstyp der diabetischen Nervenschädigung - besprochen. Weiterhin wurden Probleme der auch heute noch unterdiagnostizierten und differenzialdiagnostisch schwierigen diabetischen Gastroenteropathie behandelt. Beiträge zum apoplektischen Insult als Beispiel der bei Diabetespatienten gehäuft vorkommenden Schädigungen des zentralen Nervensystems und den ebenfalls vermehrt auftretenden Schlafstörungen konnten die Beziehungen zu den Komorbiditäten erweitern. Hier besteht ein kompliziertes Zusammenwirken von Neuropathiesymptomen, autonomer Regulation des Respirationstrakts, metabolisch-endokrinen Störungen insbesondere bei der Adipositas und psychischen Einflussgrößen. Die Zusammenhänge von Schlafstörung, Übergewicht und Schweregrad der Glukosetoleranzstörung wurden neuerdings durch eine große Querschnittsanalyse mit 2588 Teilnehmern („Sleep Heart Health Study“) bestätigt (Diabetes Care 2008, 31:1001-1006), wobei das obstruktive Schlafapnoesyndrom (OSAS) im Vordergrund steht („Sleep AHEAD Research
Group“, Diabetes Care 2009, 32:10171019).

\section{( Psychopathologische Manifestationen des Diabetes bilden einen Schwerpunkt dieses Sonderheftes}

Da weitere anstehende Themenkreise und Vorschläge nicht in einem einzelnen Sonderheft aufgenommen werden konnten, hat sich die Schriftleitung zusammen mit dem Redaktionsteam des Springer-Verlags entschlossen, ein zweites Heft mit dem Leitthema „Diabetische Neuropathie“ herauszugeben. In diesem zweiten Themenheft bilden eher medizinischwissenschaftliche Grundlagen und die in neuerer Zeit immer mehr beachteten psychopathologischen Krankheitsmanifestationen bei Diabetes mellitus, wie sie insbesondere auch in der Schmerztherapie von Bedeutung sind, den Schwerpunkt.

Die Neuropathie nimmt wegen ihrer multifaktoriellen, durch ein komplexes Zusammenspiel metabolischer und vaskulärer Ursachen gekennzeichneten $\mathrm{Pa}$ thogenese bei den diabetischen Langzeitkomplikationen eine Sonderstellung ein. In der Literatur und in großen Verlaufsstudien wird sie, soweit untersucht, wegen den neuronalen mikrovaskulären Schädigungen - analog zu Retino- und Nephropathie - häufig zu den mikroangiopathischen Komplikationen gezählt. Die seit Langem bekannte Glykierung von Proteinen bildet hier offenbar ein grundlegendes pathogenetisches Grundprinzip, das von einem der besten Kenner auf die- sem Gebiet in seinem Beitrag in hervorragender Weise dargestellt wird.

Nach wie vor gilt grundsätzlich die Aussage, dass die Verordnung einer schmerzwirksamen Medikation individuell unter Berücksichtigung der Symptomatik sowie der medikamentösen Kontraindikationen und Nebenwirkungen erfolgen muss (Leitlinien der Deutschen Gesellschaft für Neurologie, 4. Aufl., Thieme, Stuttgart, 2008). Algorithmen zur symptomatischen Schmerzmodulation wurden vorgeschlagen, sind jedoch nicht validiert. Trotz vielfältiger pharmakologischer Entwicklungen und Interventionen ist mit Ausnahme einer möglichst normnahen Blutzuckereinstellung auch heute keine allgemein anerkannte, pathogenetisch wirksame Therapie der diabetischen Neuropathie möglich.

\section{Bislang ist eine einheitliche, symptomorientierte Behandlung neuropathischer Schmerzen nicht möglich}

Nach wie vor klafft also eine Lücke zwischen unserem klinisch-therapeutischen Vorgehen und den pathogenetischen Erklärungsmöglichkeiten, die insbesondere auch die oftmals schwierige Therapie der schmerzhaften Neuropathie betrifft. Wegen der komplexen Mechanismen der Schmerzentstehung, den schwierigen allgemein gültigen Zuordnungsmöglichkeiten neuropathischer Schmerzen zu neuronalen Störungen und der komplizierten Wirkungen schmerzwirksamer Medikamente auf Ionenkanäle und Re- 
zeptorproteine sowie nicht zuletzt wegen der Komorbiditäten ist eine einheitliche, symptomorientierte Behandlung neuropathischer Schmerzen nicht möglich. Die Schmerzbehandlung muss also nach wie vor auf klinisch-empirischer Grundlage erfolgen (Einzelheiten siehe Beitrag Maag, Stengel, Baron in: Der Diabetologe 2006, 2:104-113). Im vorliegenden Themenheft wird der aktuelle Standard der Schmerzentstehung und seine Therapiemöglichkeit am Tiermodell und beim Menschen aufgezeigt und insbesondere auf die Limitationen von derzeit möglichen tierexperimentellen Untersuchungen hingewiesen.

Die Verbindung von den klassischen neuronalen Funktionsstörungen bei diabetischer Neuropathie zu den heute immer mehr beachteten Komorbiditäten und zentralnervösen Störungen wird schließlich in dem Beitrag zu „Depression, Angst und Schlafstörungen bei diabetischer Neuropathie" hergestellt. Hier hat eine aktuell publizierte große Querschnittsstudie bei 1456 Menschen mit Diabetes gezeigt, dass mit validierten Fragebögen milde bis schwere Angst- und Depressionssymptome bei 32\% bzw. 22,4\% der untersuchten Patienten mit Diabetes diagnostiziert werden konnten, wobei Diabeteskomplikationen neben anderen Ursachen signifikante Einflussfaktoren waren (Diabet Med 2009, 26:153-161). Symptome einer diabetischen Neuropathie, insbesondere auch Schmerzen, können Teilursache dieser Komorbiditäten sein und werden deshalb in bereits vorhandene und zukünftige Leitlinien einbezogen (Praxis-Leitlinien der DDG, Diabetologie 2008, 3 Suppl. 2:168-174; Nationale Versorgungsleitlinie „Diabetische Neuropathie“ 2009/2010, in Vorbereitung). Zusätzlich werden die bereits im ersten Schwerpunktheft abgehandelten klinischen Aspekte der mit Diabetes mellitus und insbesondere mit der diabetischen Neuropathie assoziierten Schlafstörungen im Hinblick auf eine präzisere Diagnostik erweitert.

Ganz aktuell kommt bei den Komorbiditäten zusätzlich die Demenzerkrankung mit ins Spiel. So konnte in einer neunjährigen Verlaufsstudie bei 1248 über 75 Jahre alten, zunächst demenzfreien Personen in Abhängigkeit vom Schweregrad des Diabetes und vom Diagnosezeitpunkt etwa dreimal so häufig eine Demenz - vorwiegend vom Alzheimer-Typ - im Vergleich zu den Patienten, die keinen Diabetes entwickelten, beobachtet werden (Diabetologia 2009, 52:1031-1039).

\section{จ Aktuelle Forschungen zur Proteinglykierung lassen für die Zukunft neue, ursächlich wirksame Therapieansätze erwarten}

Es war Ziel der beiden Schwerpunkthefte „Diabetische Neuropathie I und II“, das insbesondere bei dieser Krankheitskomplikation relevante große, interdisziplinäre medizinische und wissenschaftliche Spektrum aufzuzeigen, das für die Diagnostik und Therapie dieses facettenreichen Krankheitsbildes in Betracht gezogen werden muss. Aktuelle Forschungen zur Proteinglykierung als allgemeines Schädigungsprinzip haben zu neuen pathogenetischen Erkenntnissen der diabetischen Neuropathie geführt und werden wohl in Zukunft zur Entwicklung neuer, ursächlich wirksamer Therapieansätze beitragen. Weiterhin werden heute zunehmend bei Diabetes Typ 1 und 2 Beziehungen neurophysiologischer (z. B. kognitiver) Störungen, den oben genannten Komorbiditäten und peripher-neurologischen Dysfunktionen im Zusammenhang mit zentralnervösen morphologischen Veränderungen untersucht, um in Zukunft bei Menschen mit Diabetes mellitus Zusammenhänge im Hinblick auf individuelle Schädigungen und Beeinträchtigungen besser erkennen zu können.

\section{Hase bede}

M. Haslbeck

\section{Korrespondenzadresse}

Prof. Dr. M. Haslbeck

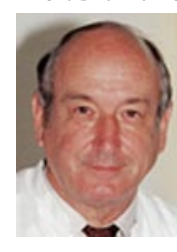

Forschergruppe Diabetes e. V. am Helmholtz-Zentrum München-Neuherberg Ingolstädter Str. 1, 85764 Neuherberg Manfred.Haslbeck@lrz. uni-muenchen.de

\section{Positive Wirkungen von Kaffee auf die Gesundheit}

Welche Auswirkungen der regelmäßige Genuss von Kaffee, einem der beliebtesten Getränke in Deutschland, auf die Gesundheit beziehungsweise das Krankheitsrisiko besitzt, ist eine interessante und daher wissenschaftlich intensiv untersuchte Fragestellung. Die Ergebnisse umfangreicher epidemiologischer Studien scheinen die Zusammenhänge zwischen Kaffeekonsum und Gesundheit erhellen zu können. Das Deutsche Grüne Kreuz e.V. in Marburg hat dazu das Ärzte-Merkblatt „Präventionsmedizinische Aspekte des Kaffeekonsums" herausgegeben, das einen Überblick über die aktuellen Erkenntnisse zu den präventiven Wirkungen von Kaffee auf unterschiedliche Krankheitsbilder gibt. In einer Übersichtsarbeit wurden zahlreiche Studien aus den vergangenen zehn Jahren ausgewertet. Die Schwerpunkte wurden dabei auf kardiovaskuläre Krankheiten, Typ-2-Diabetes, Tumorerkrankungen sowie die kognitiven Fähigkeiten und die allgemeine Mortalität gelegt. Der Autor kommt zu der Schlussfolgerung, dass sich die Sichtweise auf den Kaffee deutlich gewandelt habe. Während in der Vergangenheit häufig vor einem Kaffeekonsum gewarnt wurde, zeigten nun die vorliegenden Studiendaten, dass diese Ansicht einer Revision unterzogen werden müsse. Der Genuss von Kaffee könne offensichtlich bestimmte Krankheitsrisiken vermindern und besitze daher präventionsmedizinische Relevanz. Das ÄrzteMerkblatt kann beim Deutschen Grünen Kreuz in Marburg bestellt werden.

Die Merkblätter stehen zudem unter www.kaffee-wirkungen.de zum Download bereit.

Quelle:

Deutsches Grünes Kreuz www.dgk.de 